\title{
Hydrogen production with holes: what we learn from operando studies
}

Artur Braun, Kelebogile Maabong, Mmantsae M. Diale, and Rita Toth

Using $x$-ray spectroscopy to analyze a photoelectrochemical cell during water oxidation and hydrogen formation sheds light on the physics and chemistry of photoelectrodes.

As we become more aware of the limited amount of energy available from traditional sources, we are increasingly turning to solar power as a viable alternative. ${ }^{1,2}$ Of the total worldwide energy consumption, $20 \%$ is electrical, with an increasing share being produced by photovoltaics. Scientists, engineers, technologists, and investors are now working towards a renewable alternative for the remaining $80 \%$, which is currently obtained from fossil fuels, nuclear fuels, and biomass. ${ }^{3-5}$

Photoelectrochemical cells (PECs), which use sunlight to convert water into solar-hydrogen fuel, represent one route to achieving a renewable energy source. PECs are based on semiconductor photoelectrodes, ${ }^{6}$ but their principles of energy conversion and storage are analogous to photosynthesis. The photoelectrodes within PECs are comprised of two electrodes. At least one contains a light absorber (which is applied as a coating on a transparent conducting oxide, TCO) and one has an electrocatalytic surface (e.g., an aqueous-electrolyte coating). When light strikes the absorber, photoelectrons and holes are created. The electrons then migrate through the TCO, which acts as a current collector, and enter the electric circuit. The holes diffuse to the electrode surface, where they chemically react with water molecules and cause them to electrochemically split into oxygen gas. This gas evolves at the photoanode and can be collected in a container for any potential further use. Protons migrate through the electrolyte to the counter electrode, where they combine with electrons to form hydrogen gas, which is collected as fuel.

We have designed a PEC reactor (a prototype of which is shown in Figure 1 that has a large $(10 \times 10 \mathrm{~cm})$ iron oxide

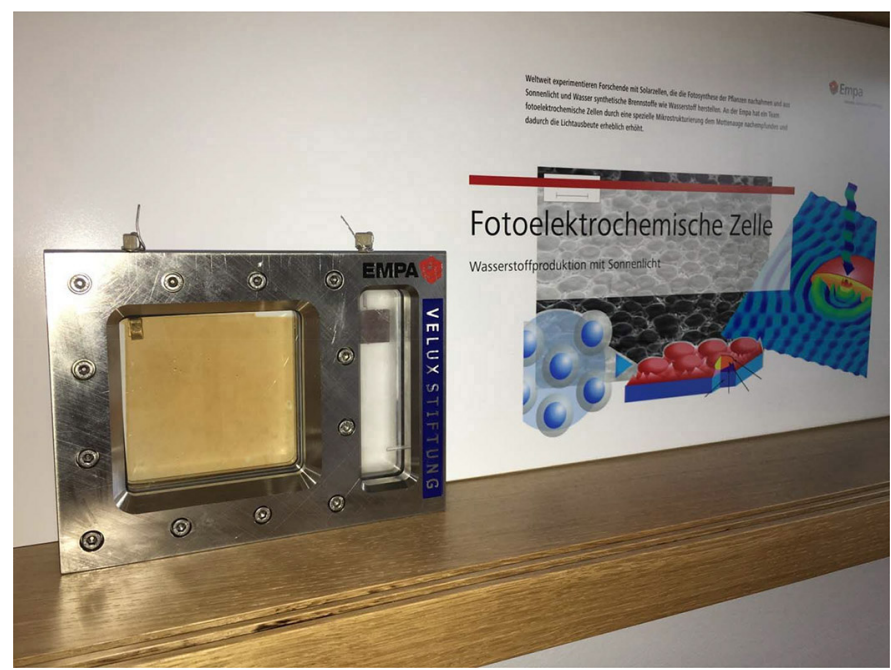

Figure 1. The photoelectrochemical cell (PEC) reactor prototype. The device has an active area of $100 \mathrm{~cm}^{2}$ and is comprised of glass coated with an iron-oxide photoelectrode. The design incorporates an oxygen gas outlet (top left). The white compartment on the right of the device holds the platinum counter electrode for hydrogen gas evolution and collection. One molar mass of potassium hydroxide, acting as the electrolyte, is supplied continuously.

photoanode (applied as a coating on glass, i.e., the TCO) in a sealed steel frame filled with an electrolyte ( 1 mole of potassium hydroxide). The design of our units means they can be arranged in large clusters, like photovoltaic panels, and could therefore enable the development of a decentralized, scalable fuel-production facility (i.e., which could improve the resilience of human settlements). ${ }^{7}$ Moreover, by using a form of x-ray spectroscopy, we can measure processes that occur in our device as they happen (i.e., 'operando' measurements). In this way, we are able to unveil processes and characteristics that are otherwise extremely difficult to investigate. ${ }^{8}$ 

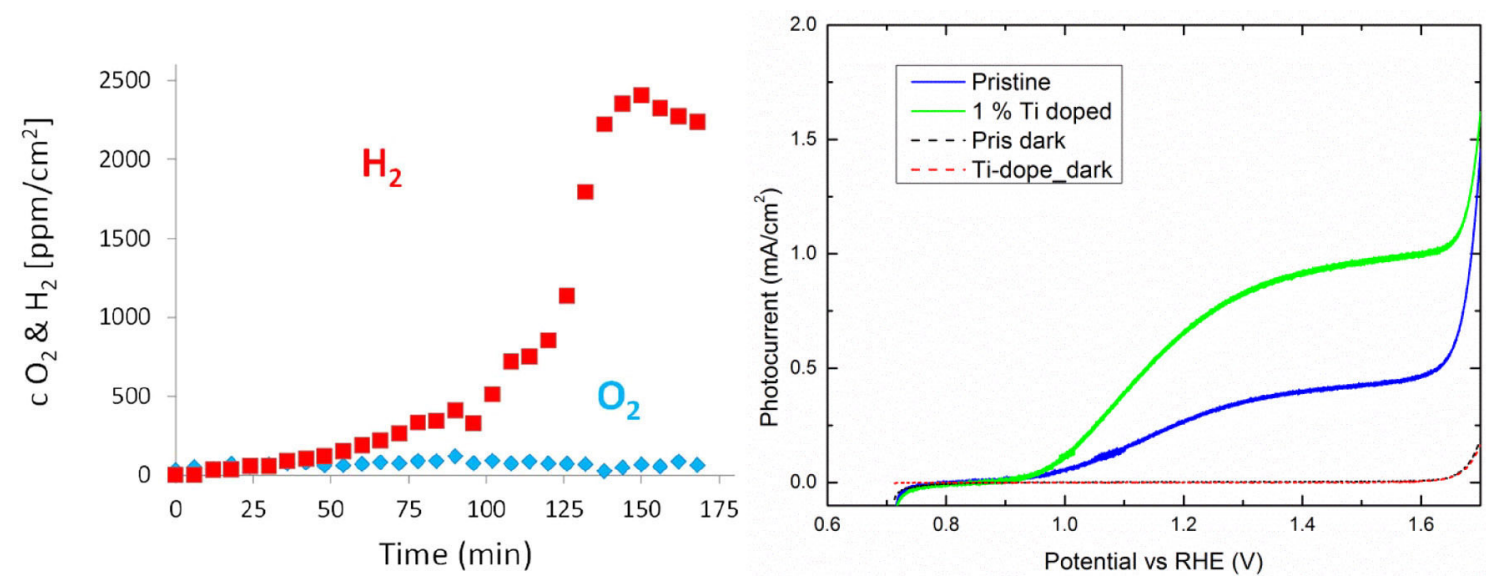

Figure 2. Left: Transient concentration (c) of evolved oxygen, $\mathrm{O}_{2}$ (blue diamonds), and hydrogen, $\mathrm{H}_{2}$ (red squares), during water splitting in a PEC under 1.5AM illumination. Right: Evolution of light-current density in pristine (blue) and titanium-doped (green) iron-oxide photoelectrodes under illumination conditions. Dark currents are represented by the red and black dashed lines. RHE: Reversible hydrogen electrode.
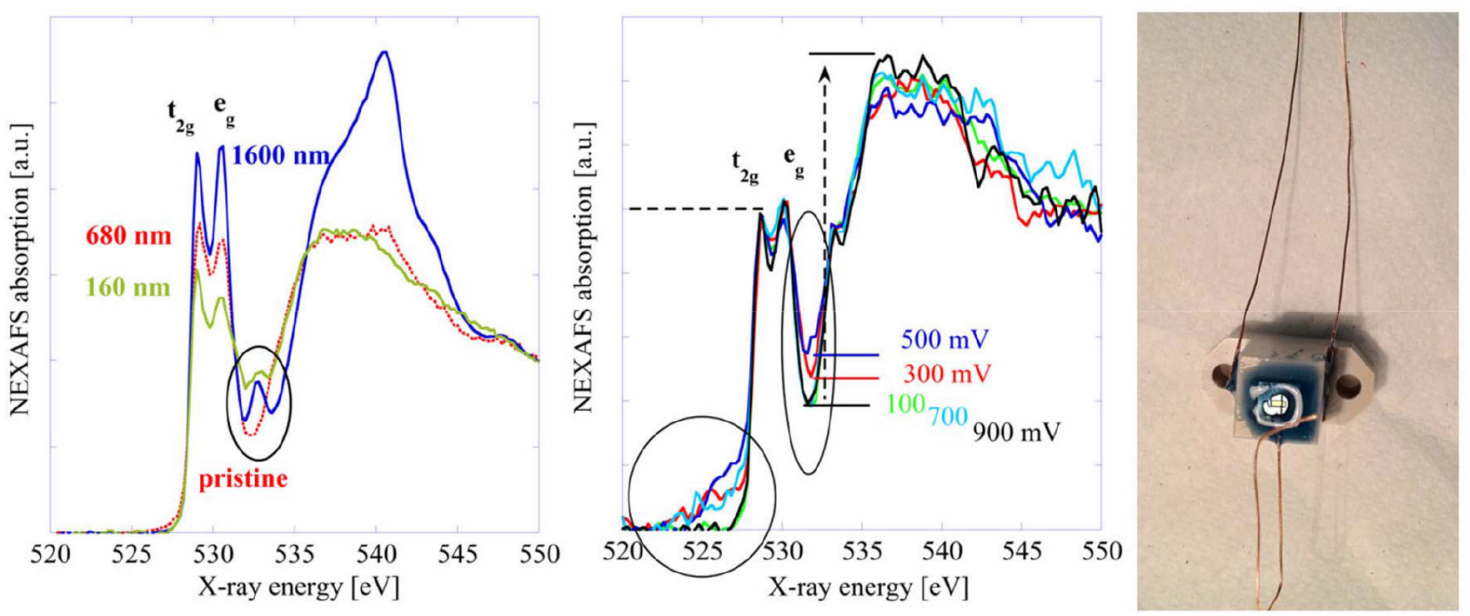

Figure 3. Left: Three near-edge $x$-ray absorption fine structure (NEXAFS) spectra (for the oxygen 1s orbital), obtained at $600 m V$ operation and under vacuum at the BESSY II synchrotron facility in Germany. The spectra are for a 680nm-thick pristine (red spectrum) and two Ag+/AgCl (i.e., silver/silver chloride) anodized hematite photoelectrodes, with thicknesses of 160 and 1600nm (green and blue spectra, respectively). Middle panel: Five NEXAFS spectra obtained for a 30nm-thick hematite film deposited in a miniature in situ cell (shown on the right). e, t: Doubly and triply degenerate-level orbitals, respectively. g: Odd numbers. a.u.: Arbitrary units. Reprinted with permission from Elsevier. ${ }^{9}$

The processes that are relevant for the production of hydrogen in a PEC are among the most complex in physical chemistry, and traditionally, electrochemical, electroanalytical, and optical methods have been employed as analytical tools in this field. Figure 2 shows the evolution of the current density in our prototype cell as a function of external bias from a hematite photoelectrode, under both dark and illuminated (AM1.5, i.e., an air mass coefficient of 1.5) conditions. To carry out this experiment, we used a potentiostat. We also used a gas chromatograph to measure the evolution of the hydrogen and oxygen. Although the efficacy of the process and the reactor can be assessed using these methods, they do not reveal many details about the relationships between synthesis, processing, structure, and functional performance in PECs. This is partly because of the difficulties associated with observing the processes that occur between the electrode and electrolyte during energy conversion. For such studies, one must employ operando analytical methods.

Continued on next page 
To understand our system at the molecular level, we therefore used near-edge $x$-ray absorption fine structure spectroscopy (NEXAFS) to investigate the photoelectrodes ex situ and under vacuum. We found that a DC bias potential of $600 \mathrm{mV}$ led to the formation of a surface layer with an oxy-hydroxy structure (see Figure 3 left). ${ }^{10}$ This iron oxide-hydroxide layer, formed as a consequence of anodic oxidation, is evidenced by the appearance of a peak at $532 \mathrm{eV}$. The process also leads to hole doping, as revealed by the shift of the resonant photoemission valenceband spectra towards the Fermi level. Our results suggest that the extent of hole doping depends on bias potential and bias duration. ${ }^{11,12}$

We conducted NEXAFS during water splitting (i.e., operando) in a microreactor (see Figure 3 , right) and found hole states in the pre-edge of the resultant spectra. We were able to switch these states on/off by applying/removing the minimum bias potential and switching the solar simulator light on/off (see Figure 3. middle). We thus obtained the first experimental proof of the PEC theory at the molecular scale with x-ray spectroscopy. ${ }^{13} \mathrm{We}$ also made operando measurements of the spectra under an electrochemical bias of $100-900 \mathrm{mV}$ and an illumination of $1.5 \mathrm{AM}$ (i.e., simulating solar light conditions). In addition to the aforementioned changes, we obtained a new spectral weight from transient holes at $526 \mathrm{eV}$. These transient holes oxidize the water molecules. The resulting breakage of the hydrogen bonds among the water molecules is indicated by a slight spectral shift at $535 \mathrm{eV}$.

In summary, we have developed PECs that can be arranged in large clusters for efficient energy collection. In addition, we have made operando measurements of our PECs (using NEXAFS), and have observed a number of device characteristics that are extremely difficult or impossible to obtain using other methods. With our results we are able to predict which process and operation parameters will give rise to specific physical and chemical changes. For instance, the detection of transient electron holes at $526 \mathrm{eV}$ in the NEXAFS spectra (see Figure 3 . middle) would not be possible without operando measurements. The same is true for the measurement of the hydrogen-bond-breaking peak at $535 \mathrm{eV}$. The existence of two different energetic electron holes was predicted ${ }^{14}$ almost 30 years ago from electrochemical and optical spectroscopy investigations (i.e., based on a disproportionation of iron), but only the NEXAFS results reported here provide direct experimental evidence for the second electron hole. Progress in the analytical sciences is helping us to understand the complexity of PEC reactors, and we are now preparing to build actual large PEC reactors to engage with energy-systems engineers, as well as to advance civil and landscape engineering.

\section{Author Information}

\section{Artur Braun and Rita Toth \\ Empa \\ Duebendorf, Switzerland}

Artur Braun is an expert in the materials science of electrochemical systems. His research group at Empa develops x-ray and neutron operando methods for batteries, fuel cells, and solar cells. $\mathrm{He}$ is a Marie Curie Fellow, and Foreign Policy Magazine has listed him as a Leading Global Thinker of 2014.

Rita Toth is an expert in physical chemistry and her research focuses on self-organization and pattern formation in dynamic systems. At Empa, she develops online gas chromatography methods for electrochemical energy conversion and storage. She is a Marie Heim-Vögtlin Fellow, and Foreign Policy Magazine has listed her as a Leading Global Thinker of 2014.

\section{Kelebogile Maabong \\ University of Botswana \\ Gaborone, Botswana}

Kelebogile Maabong is a physicist and former lecturer at the University of Botswana. She is currently pursuing a PhD at the University of Pretoria, South Africa. Her research focuses on understanding the electronic band structure of semiconductor metal oxides by means of symmetry and group-theoretical methods towards energy conversion.

\section{Mmantsae M. Diale \\ University of Pretoria \\ Pretoria, South Africa}

Mmantsae Diale is a physics professor in the electrical characterization of surfaces, interfaces, and defects in semiconductors. Her research group at the University of Pretoria is focused on bio and hybrid organic and inorganic materials and devices for solar energy conversion.

\section{References}

1. A. Braun, N. Gaillard, E. L. Miller, and H. Wang, Advanced materials and structures for solar fuels: introduction, J. Mater. Res. 31, pp. 1545-1546, 2016.

2. A. Thapper, S. Styring, G. Saracco, A. W. Rutherford, B. Robert, A. Magnuson, W. Lubitz, et al., Artificial photosynthesis for solar fuels - an evolving research field within AMPEA, a joint programme of the European Energy Research Alliance, Green 3, pp. 4357, 2013.

3. T. Randall, Wind and solar are crushing fossil fuels, Bloomberg Europe, 2016. https://www.bloomberg.com/news/articles/2016-04-06/wind-and-solar-arecrushing-fossil-fuels Accessed 02 April 2017. 
4. R. Swenson, The solarevolution: much more with way less, right now - the disruptive shift to renewables, Energies 9, p. 676, 2016.

5. D. Scholten and R. Bosman, The geopolitics of renewables; exploring the political implications of renewable energy systems, Technol. Forecast. Soc. Change 103, pp. 273283, 2016.

6. M. Grätzel, Photoelectrochemical cells, Nature 414, pp. 338-344, 2001.

7. A. Braun, M. M. Diale, K. D. Maabong, and R. Toth, Safe and decentralised solar hydrogen fuel production and storage for residential building and mobility applications, $\mathbf{6}^{\text {th }}$ Int'1 Disaster Risk Conf., pp. 102-105, 2016.

8. A. Braun, X-Ray Studies on Electrochemical Systems: Synchrotron Methods for Energy Materials, p. 474, De Gruyter, 2017.

9. A. Braun, Y. Hu, F. Boudoire, D. K. Bora, D. D. Sarma, M. Grätzel, and C. M. Eggleston, The electronic, chemical and electrocatalytic processes and intermediates on iron oxide surfaces during photoelectrochemical water splitting, Catal. Today 260, pp. $72-$ 81, 2016.

10. D. K. Bora, A. Braun, S. Erat, A. K. Ariffin, R. Löhnert, K. Sivula, J. Töpfer, et al., Evolution of an oxygen near-edge $x$-ray absorption fine struction transition in the upper Hubbard band in $\alpha-\mathrm{Fe}_{2} \mathrm{O}_{3}$ upon electrochemical oxidation, J. Phys. Chem. C. 115, pp. 5619-5625, 2011.

11. A. Braun, Q. Chen, D. Flak, G. Fortunato, K. Gajda-Schrantz, M. Grätzel, T. Graule, et al., Iron resonant photoemission spectroscopy on anodized hematite points to electron hole doping during anodization, ChemPhysChem 13, pp. 2937-2944, 2012.

12. K. Gajda-Schrantz, S. Tymen, F. Boudoire, R. Toth, D. K. Bora, W. Calvet, M. Grätzel, E. C. Constable, and A. Braun, Formation of an electron hole doped film in the $\alpha-\mathrm{Fe}_{2} \mathrm{O}_{3}$ photoanode upon electrochemical oxidation, Phys. Chem. Chem. Phys. 15, pp. 1443-1451, 2013.

13. A. Braun, K. Sivula, D. K. Bora, J. Zhu, L. Zhang, M. Grätzel, J. Guo, and E. C. Constable, Direct observation of two electron holes in a hematite photoanode during photoelectrochemical water splitting, J. Phys. Chem. C. 116, pp. 16870-16875, 2012.

14. J. H. Kennedy and K. W. Frese Jr., Photooxidation of water at $\alpha-\mathrm{Fe}_{2} \mathrm{O}_{3}$ electrodes, J. Electrochem. Soc. 125, pp. 709-714, 1978. 